\title{
Drag reduction via Turbulent Boundary Layer Flow Control
}

\author{
Adel Abbas ${ }^{1}$, Gabriel Bugeda ${ }^{2,3}$, Esteban Ferrer ${ }^{1,4}$, Song FU ${ }^{5}$, Jacques Periaux ${ }^{2}$, \\ Jordi Pons-Prats ${ }^{2}$, Eusebio Valero ${ }^{1,4}$, Yao ZHENG ${ }^{6}$ \\ ${ }^{\prime}$ ETSIAE-UPM - School of Aeronautics, Universidad Politécnica de Madrid, Pza. Cardenal Cisneros 3, Madrid, E-28040, Spain \\ ${ }^{2}$ CIMNE, Gran Capità s/n, Campus Nord UPC, 08034 Barcelona, Spain \\ ${ }^{3}$ UPC Barcelona Tech, Civil and Environmental Department, Gran Capità s/n, Campus Nord UPC, 08034 Barcelona, Spain \\ ${ }^{4}$ Centre for Computational Simulation-UPM, Scientific and Technological Park (Campus Montegancedo), 28223 Pozuelo de Alarcón, Spain \\ ${ }^{5}$ Tsinghua University, School of Aerospace Engineering, Beijing 100084, China \\ ${ }^{6}$ Zhejiang University, Faculty of Engineering, Yuquan Campus, Hangzhou, Zhejiang 310027, China
}

Received ??, 2016; accepted ??; published online ??

\begin{abstract}
Turbulent Boundary Layer Control (TBLC) for skin-friction drag reduction is a relatively new technology made possible through the advances in computational-simulation capabilities, which have improved the understanding of the flow structures of turbulence. Advances in micro-electronic technology have enabled the fabrication of active device systems capable of manipulating these structures. The combination of simulation, understanding and micro-actuation technologies offer new opportunities to significantly decrease drag, and by doing so, increase fuel efficiency of future aircraft. The literature review that follows will show that the application of active control turbulent skin-friction drag reduction is considered of prime importance by industry, even though it is still at a low Technology Readiness Level (TRL). This review presents the state of the art of different technologies oriented to the active and passive control for turbulent skin-friction drag reduction and contributes to the improvement of the actual present status of these technologies.
\end{abstract}

Turbulent boundary layer flow control, drag reduction

PACS number(s): 47.27.Rc, 47.85.L, 47.85.1b

Citation: ??

\section{Introduction}

Modern engineering and technological developments are becoming increasingly driven by the need to boost efficiency and to reduce environmental impact. Climate change has become one of the major motivations for improvements in different technological areas, in aeronautics in particular. It is highly probable that the above concerns will translate into more complex regulatory actions over the next coming

${ }^{2}$ Corresponding author (email: bugeda@cimne.upc.edu) years. This new interest in designing friendly environment aircraft is a major challenge for aircraft manufacturers. The need for greatly reduced emissions, particularly of greenhouse gases such as carbon dioxide and nitrogen oxides, will establish new paradigms in aircraft design. Three main approaches to achieving this reduction can be identified:

1) Improved aerodynamic design of airframes.

2) Lighter aircraft configurations,

3) Improved efficiency of aero-engines,

The efficient design of aero-engines can make a significant contribution to reduce the produced level of pollution. However, emissions can only be significantly reduced 
ultimately by burning less fuel, which can be achieved by reducing the drag generated during flight.

With respect to the problem of drag reduction in aeronautical applications, two different aspects must be considered. First, most of the flows in aeronautical applications involve high Reynolds numbers (high speed) during flight regime conditions, and therefore exhibit turbulent flow characteristics. Second, the main component of the total drag being the viscous (mostly skin-friction) drag (Figure 1), any reduction strategy will consider the region close to the wall where the friction drag is generated. In this context, a flow control methodology targeting turbulent boundary layers (TBL) is the most obvious option to achieve a significant skin-friction-drag reduction and ultimately to reduce emissions. In spite of the extensive amount of investigation and work accumulated over the years, the study of turbulent boundary layers is still an active area of research; recently feed with rising interest in drag reduction and new clean technologies.

As is illustrated in Figure 1, almost 50\% of total drag is due to the viscous drag, which is directly related to the friction drag of the aircraft caused by the interaction of the turbulent boundary layer flow with the aircraft surface. Studies

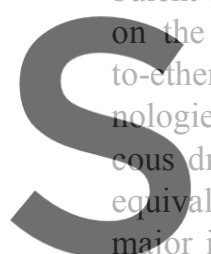

aircraft and turbulent

with developments of a

can effectively reduce

rag [1] (if the actuation pov

lent to about $15 \%$ of the to

implications to fuel consumption

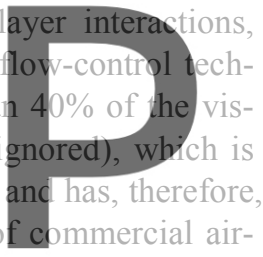

craft, even if a small proportion of this reduction level is

Register for free at https//www.scipedia.com to $\begin{array}{cc}\text { Drag } & \text { Technology } \\ \text { Break-down } & \text { Opportunities }\end{array}$
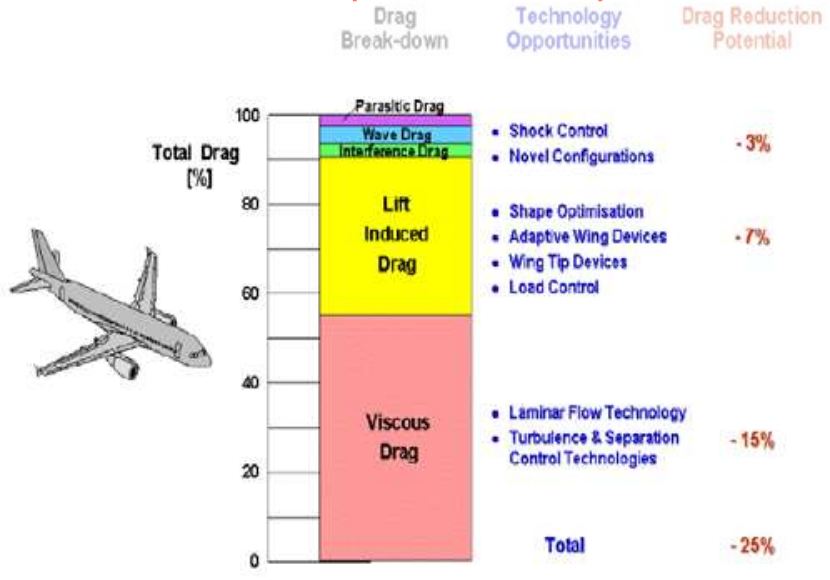

Figure 1 Concept of aircraft drag forces and drag reduction potential analysis.

The exploration of drag-reduction technologies is not new, initialized in the early 70', driven by the oil crisis. NASA Lang-ley Research Centre found that ribbed wall surfaces (passive riblets) can significantly reduce resistance, which eventually changed the traditional belief that smooth surfaces are unrivalled for low drag. Subsequently, a variety of drag-reduction methods and theories were proposed and developed or realized. However, most of the drag-reduction technologies have not yet been incorporated into modern aircraft. The difficulties of applying control devices in industrial conditions is mainly related to limitations in traditional manufacturing methods and to the additional energy consumption and weight associated with the gross drag-reduction levels that may results in a negative net gain. More recently, new developments in micro technology and improved manufacturing processes are enabling new concepts for active and passive flow control to be realized, hence creating new opportunities for the control of turbulent boundary layer.

Turbulent skin-friction drag reduction through passive and active flow control is still at an early stage in its development. Both moving-wall and body-force control strategies have been shown to give drag reductions in both numerical and experimental studies. In fact, there are strong indications that significant drag reductions, greater than $40 \%$ in certain circumstances [2], can be achieved. However, overall efficiency remains a largely unexplored subject, and

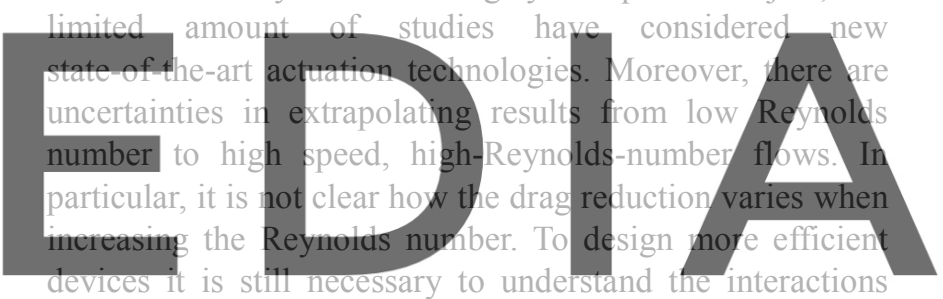

between inner and outer wall mechanisms and the role of

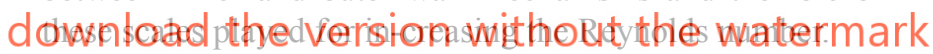

\section{Strategies for turbulent control}

From a technical point of view, the objective of turbulent drag-reduction technologies is to reduce the turbulent shear stress in the near wall region. Several DNS-based studies [3] provided direct evidence of a link between the production of Reynolds stresses and the quasi-streamwise vortical structures in the near-wall buffer layer; $10<y+<30$, where here and throughout this document "+" indicates non-dimensionalisation with respect to wall units ${ }^{1}$. Previous work [4] revealed that streamwise vortices tend to be located above and a little to the side of high skin friction regions suggesting that the interaction between these vortices and the wall is critical to the generation of wall shear stress and thus skin friction.

Recent discoveries explain that drag-reduction strate-

\footnotetext{
1 Wall units are based on the friction velocity and the kinematic viscosity.
} 
gies are potentially more efficient when generating vortices that interfere with the streak formation in the viscous inner-layer $(y+<10$ at least for low Reynolds numbers) than those directed at counteracting the vortices once formed. However, as the Reynolds number increases, the role of the outer structures may become more important. Therefore, controlling these outer structures will be necessary for aircraft application of industrial relevance, i.e. high Reynolds numbers.

In general, the overall aim and the focus of control for turbulent drag reduction are to suppress or mitigate the effect of these streamwise vortices in the buffer layer. This can either be achieved through:

1- Directly targeting existent vortices;

2- Intervention elsewhere in the turbulence cycle so as to reduce their formation in the first place.

The first is a control strategy that influences the outer layer, e.g. via a suitable body force. New research has shown that, as the Reynolds increases, the large coherent streamwise structures in the outer layer could become more important in the production of shear stress close to the wall. Outer-layer structures are relatively large (compared to those in the near wall) with a length scale of order $\sim 10 \delta, \delta$
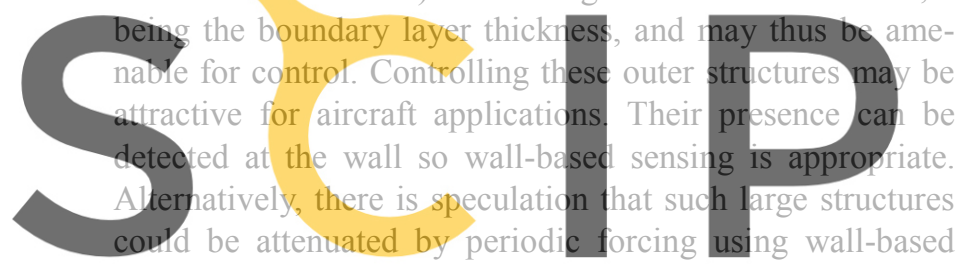

actuation

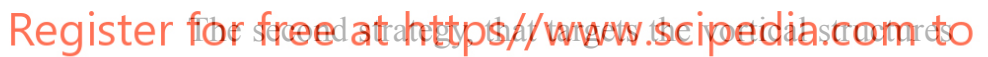
as they are generated near the wall, has been the subject of a number of recent studies, and there is evidence that the most effective interaction can be obtained via the imposition of a spanwise forcing, either in the form of a wall movement or in the form of a spanwise-oriented body force.

The actuators required by both control types need to meet several requirements if the technology is to be applied to the aircraft:

- The size and response time of the actuators have to be comparable to the length and time scale typical of the flow structures to be controlled.

- The flow perturbations produced by the actuators must be large enough to be effective in the boundary layer.

The actuators must be robust in the aircraft harsh environment: low temperature, pressure fluctuations and high flow speed should not jeopardize their functioning. According to the direction of the forcing created by the actuation, one can distinguish between:
- Out-of-plane actuation, which introduces a perturbation in the direction perpendicular to the wall.

- In-plane actuation, which introduces a perturbation in the direction parallel to the wall.

From all the above, four actuation types should be considered: (1) local wall-surface deformations (mostly wavy groove), (2) moving walls, (3) jet actuators and (4) plasma actuators. These four classes of actuators devices are sketched in Figure 2.

Outer-layer structures in the turbulent boundary layer are targeted to minimize their influence on the production of shear stresses near the wall. The approach is to disrupt the development of the turbulence structure using out-of-plane body forcing by a surface mounted actuator. This approach can also be used to minimize the influence of the outer structures on the production of shear stress near the wall; for example by means of periodic wall-normal body force to modulate the flow. Of course, by trying to influence the outer structures through surface mounted actuation, it is appreciated that the inner structures in proximity to the surface actuator will also be directly affected by it. This influence requires further study and efforts towards a better understanding.

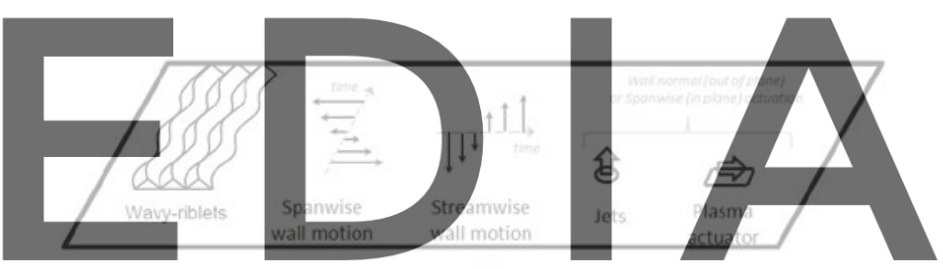

download the version without the watermark

Figure 2 Sketch of drag reduction devices: geometry modification (riblets), spanwise forcing at the wall, jet actuators and plasma actuators.

Other types of actuators aim at modifying the near-wall structures by introducing disturbances near the wall to interrupt or alter the turbulence-production cycle. Since these structures are small relative to the outer structures, it becomes difficult to disrupt them individually, and an open-loop approach becomes almost mandatory. Actuation strategies of this type include moving walls that apply a time-periodic spanwise forcing. The frequency of the wall movement can be determined by means of mean or local flow variables. Another example of mechanisms targeting inner structures is found in jets or plasma actuators. The former mimic normal-to-wall deformation, whilst the latter produce effects akin to in-plane wall deformations.

A more detailed review and expected advances beyond the state of the art of these actuators, how the boundary layer is modified and the expectations on drag reduction is given below. 


\section{State of the Art in actuation technologies for turbulent drag reduction}

\subsection{Change of wall geometry}

The planar geometry of the wall may be changed by active surface deformation, via devices that have an influence on the turbulent boundary layer by creating dynamic modifications of the surface shape such as bumps or dimples. They create an out-of plane flow perturbation to influence coherent structures in the flow, such as high-speed streaks and quasi-streamwise vortices (QSV). The characteristic dimension and response time of the actuators for the feedback control of turbulence must correspond to the scale of these structures. According to Kasagi et al [5,6], QSVs have a typical diameter $\mathrm{d}^{+}=30$ and frequency $\mathrm{f}^{+}=0.01$, which corresponds to a dimension below $1 \mathrm{~mm}$ and a response time faster as $1 \mathrm{~ms}$ for aircraft turbulent flow-control applications.

\section{Passive surface-shape} advantages of not requiring auxiliary actuation equipment. In the 1970s, many companies in the United States, Britain, Germany and other countries, began to engage in the usage of this technology to reduce the viscous drag turbulent boundary layer. Riblets (see Figure 3) are the passike technique which is nearest to applicat reviews have been given by Visw McLean [8] Laboratory studies reduce viscous drag by about $8 \%$. implemented in practical ap 3) are th
wanath [7]
The tech
pplications.

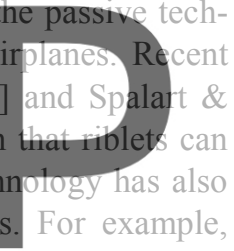
part of an Airbus A320 was covered by a striped film, resulting in a $1 \%$ to $2 \%$ reduction in fuel consumption. Flight

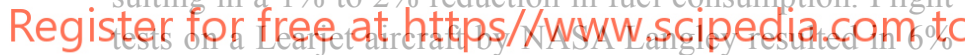
reduction in drag.

Studies on the local wall-surface deformation technology and related mechanisms have been carried out for nearly 50 years. Walsh \& Weinstein [9] investigated the relationship among the ridge structure shape, size and drag reduction. Bechert and coworkers [10] reported wind-tunnel studies for ridged-surface drag-reduction that suggested that the ridges could restrict the flow of spanwise vortex motion. Gallagher found that the grooved surface causes an effective increase in the thickness of the viscous sublayer, which plays an important role in drag. However, there are no generally accepted conclusions on the precise mechanisms at play. One of the reasons for this is the smaller size of the trend which renders it difficult the acquisition of internal flow information. Luchini [11] suggested that the difference between the longitudinal and transversal protrusion heights explains the drag reduction. More recently, Garcia-Majoral and Jiménez [12] demonstrated that riblet performance correlates better with the cross-sectional area of the riblets profile. At the same time, several optimizations of the stream- wise riblets have been proposed: for example Sasamori et al [13] tested recently the sinusoidal-riblets concepts.
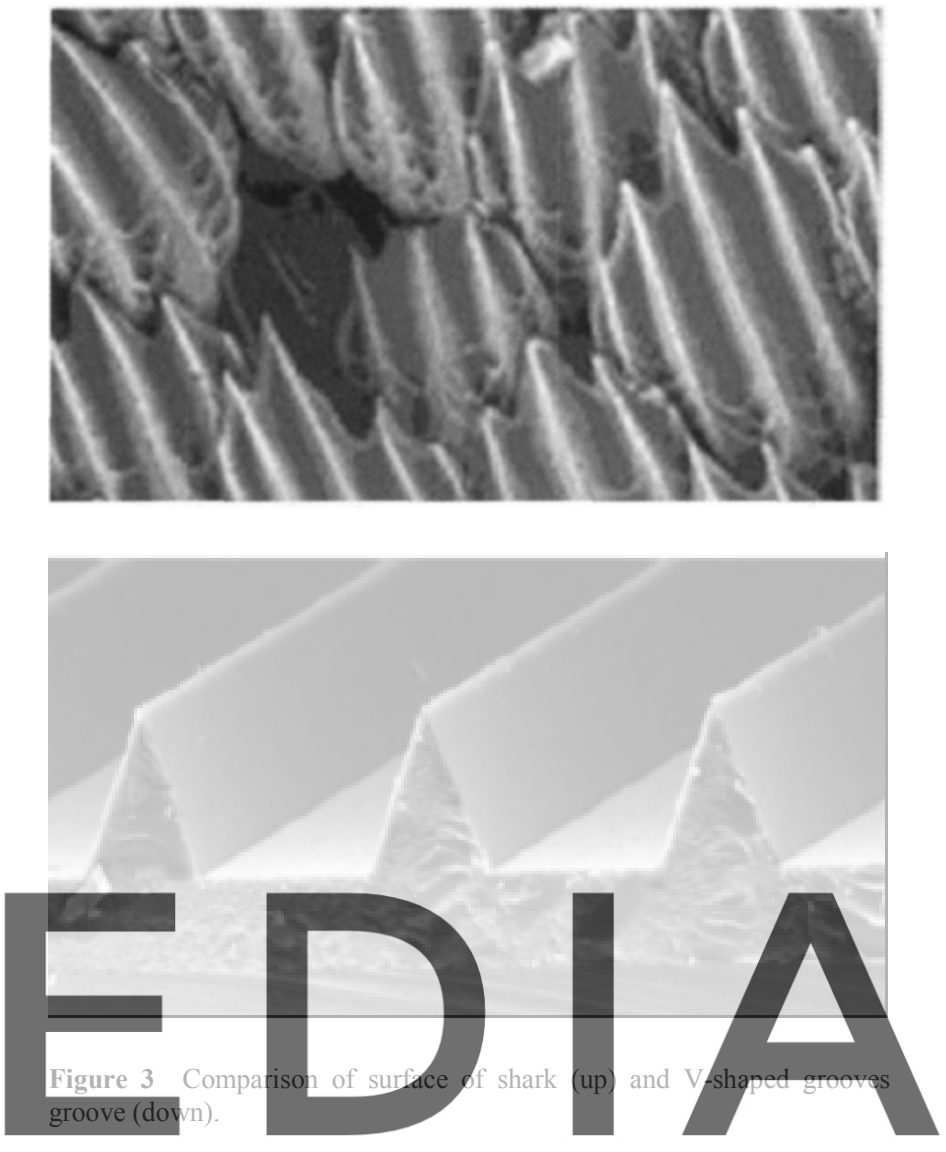

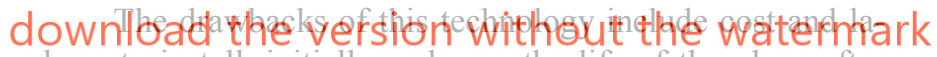
bour to install, initially and over the life of the plane after erosion take place and/or the new surface peel off. New materials or methods are almost certain, sooner or later, to bring this technology into use.

In summary, local wall surface deformation technology such as the ridged surface for drag reduction and the physical mechanisms at play require further study.

\subsection{Moving walls.}

Any method that aims to reduce turbulent friction drag has, in principle, to weaken the streaky near-wall structures and thus the wall-normal motions (ejections/sweeps) that cause wall-normal momentum exchange between inner/outer structures. The imposition of wall-parallel unsteady transverse shear is seen as one effective way of doing so, in so far as it results in turbulent-reductions of $40 \%$ and higher in carefully chosen actuation scenarios. The detailed process by which this unsteady shear interacts with the streaks is complex, and is the subject of several published papers. The main elements at play are the destabilisa- 
tion of the streaks by a continuous change in the direction of the vectorial strain in the buffer layer, a destabilization of the regeneration process following streak weakening within parts of the unsteady cycle (i.e. a lowering of turbulence production) and the unsteady transfer of wall-normal vorticity (representing streak strength) to the spanwise direction.

There are many ways of applying the unsteady actuation - streamwise-homogenous spanwise motion, streamwise travelling waves and spanwise travelling waves being the principal methods. Common to all these is that the unsteady strain has to be confined to the viscous sublayer, as the penetration of the strain into the turbulent region leads to addition strain-induced turbulent production, which counteracts the streak-weakening effect in the viscous sublayer. This dictates a relatively high frequency of actuation, the most effective being around a wall-scaled period of $\mathrm{T}^{+}=100$ (in channel flow).

Extensive DNS simulations by Quadrio [14,15] and his colleagues, the large majority being at low Reynolds numbers, show that wave-like spanwise motion, if carefully chosen in terms of frequency and wave length, is much more effective, in terms of net-energy-saving parameters, than simple spanwise motion (without waves). From a
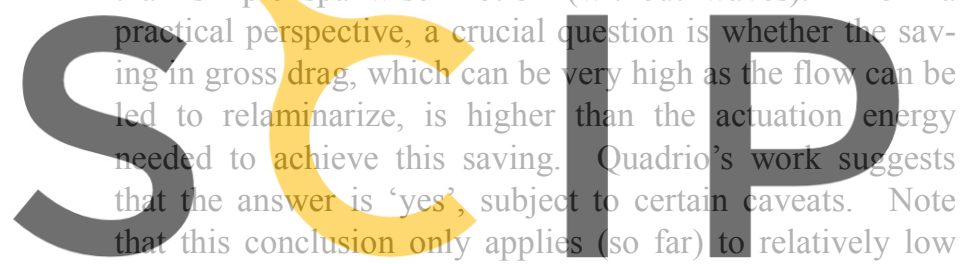

Reynolds numbers. DNS studies by Leschziner's group

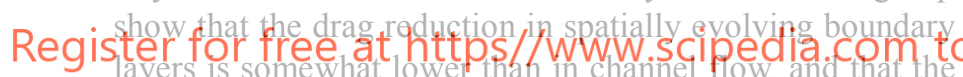

optimum actuation parameters are slightly different.

The DNS work of the groups of Leschziner [16-18] and Chung [19] (at Imperial College and Warwick University, respectively), have focused on issues somewhat different from those of Quadrio's, the latter concerned primarily with predicting the wall friction. Leschziner's work is aimed at developing a better understanding of the fundamental interactions between the spanwise forcing and the turbulent flow in the presence of purely oscillatory spanwise wall motion. More recently, these groups have examined the effects of energetic outer structures (in the log-law region) on the near-wall flow at a relatively high friction Reynolds number of 1020. Importantly, these outer structures are decoupled from the mode of near-wall actuation; i.e. these are very similar to wavy or streamwise-constant actuations. The latter observation enables the study in relatively simple actuation scenarios.

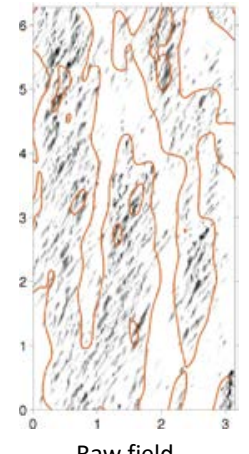

Raw field

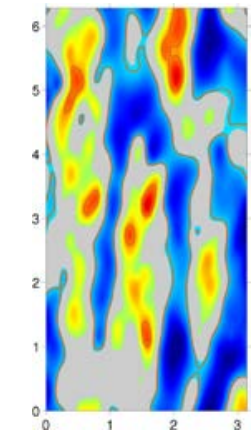

Footprint of "Super streaks"

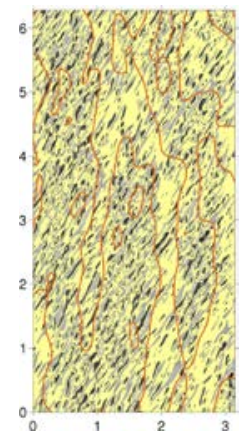

Modulated Streaks
Figure 4 Streaky structure in buffer layer of channel flow at friction Reynolds number 1020 and wall-scaled actuation period 200 (note the weakening/strengthening of steaks in different parts of the large-scale footprints).

The potential importance of large outer scales on the near-wall physics is conveyed by Figure 4, relating to a channel flow subjected to oscillatory motion at a wall-scaled period $\mathrm{T}^{+}=200$. This shows the streaky structure in a plane lying within the buffer region. The middle and right-hand plots show, respectively, the large-scale

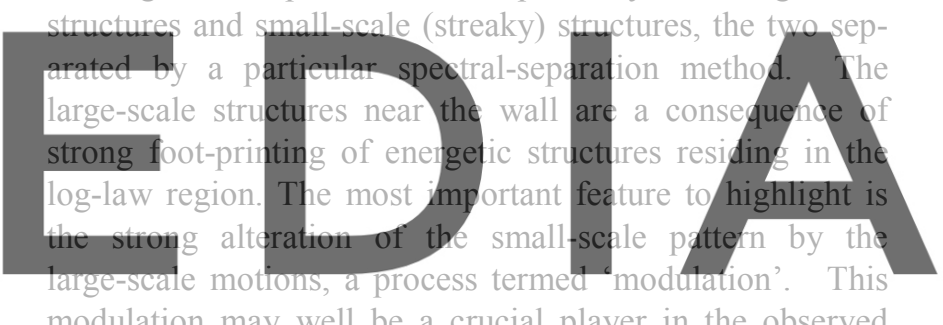

modulation may well be a crucial player in the observed

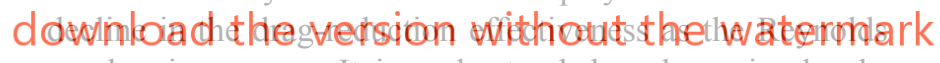
number increases. It is understood, based on simple observations of the upward shift in the log law in actuated conditions, that there should be a gentle decrease in the drag-reduction margin when increasing the Reynolds number. However, the observed dependence is much stronger, and this poses a substantial question mark on the wall of oscillatory wall actuation in high Re flows of industrial interest.

Experimental work on unactuated boundary layers by Marusic [20] and his colleagues shows that the large-scale outer motions increase in strength and energy as the Reynolds number increases, while the small-scale near-wall statistics are universal, in so far as scaling by the wall shear stress collapses the small-scale energy. This disparity of behaviour and effects has important implications to actuated conditions, and suggest that there is an urgent need to understand the effect of the large-scale on the near-wall behaviour of the small-scale steaks and in particular, their stability. 


\subsection{Jet actuators.}

These actuators modify the turbulent boundary layer by introducing variations of the flow field through orifices in the wall. Typically these operate in the out-of-plane direction. However with a correctly shaped orifice, a perturbation approximating to an in-plane body force can be introduced. One type of actuation is by means of synthetic jets. Such jets use a cavity with a membrane or a piston that alternately blows and sucks air through the orifice to secure a zero net mass flow. Alternatively, pulsed jets can be created using a pressurized air supply and an orifice that can be periodically opened and closed.

Dahm and Diez [21,22] presented a large array of 25,600 micro-actuators using the electro-kinetic principle to generate a flow displacement, potentially suitable for active sublayer control on aeronautical and hydronautical vehicles. A porous polymer matrix was used to enlarge the electro-kinetic effect produced by the displacement of ions in a micro-channel under the effect of a voltage difference. Up to $10 \mathrm{kHz}$ actuation was demonstrated with a voltage around $20 \mathrm{~V}$,

Breuer and Rathnasingham [23] built a feedback control system for the active control of the near-wall region of a

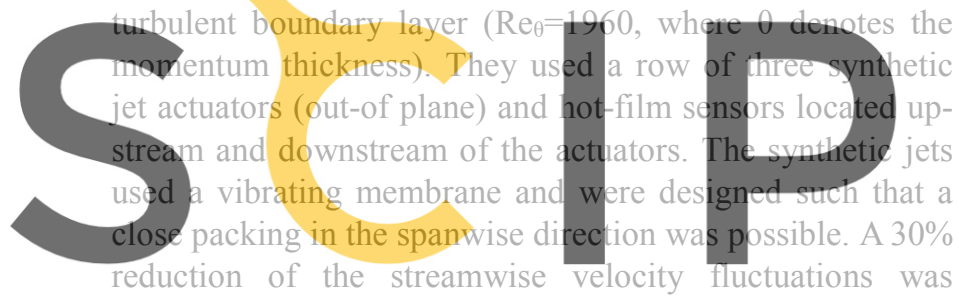
achieved.

Register for free at https//www.scipedia com to

on a turbulent flow in a rectangular channel was investigated by Iuso and Cannata [24], at a Reynoids number $\mathrm{Re}_{\tau}=180$ and a forcing frequency of $4 \mathrm{~Hz}$. Hot-wire probes measured the wall shear stress and the streamwise velocity. Turbulence attenuation up to $20 \%$ was observed.

Jet-actuator technologies have also been developed in connection with applications to separation control. These typically have a higher jet-velocity requirement compared to turbulent flow. However, the technology may still be relevant to turbulence control. Pimpin, Kasagi et al. [25,26], and Rosset et al. [27] used an electrostrictive material of polydimethylsiloxane (PDMS) as an oscillating membrane combined with compliant electrodes to produce synthetic jets. In Pimpi's work, the membrane electrodes were patterned to enhance the deflection up to $112 \mu \mathrm{m}$, which corresponds to $5.6 \%$ of the actuator diameter. The jets had a maximum velocity of $0.4 \mathrm{~m} / \mathrm{s}$ at $1.1 \mathrm{kHz}$ at a location $4 \mathrm{~mm}$ downstream from the orifice. Micro-magneto-mechanical systems (MMMS) for flow control were developed by Pernod, Merlen at al. [28,29]. A magneto-dynamic micro-valve was presented, providing high-speed jets velocities in the range of $150 \mathrm{~m} / \mathrm{s}$ with a $\mathrm{kHz}$ frequency range. Piezoelectric active diaphragms developed and tested at NASA are reported by Mane, P., Mossi, et al [30]. Jet velocities of 40 $\mathrm{m} / \mathrm{s}$ were measured but at a low frequency of $100 \mathrm{~Hz}$ and for an actuator of comparably large dimensions (88mm diameter). A composite diaphragm using Shape Memory Alloy sheet and an electromagnetic system were used by Liang et al. [31] to produce jet velocities of $190 \mathrm{~m} / \mathrm{s}$, but the frequency range was limited to $300 \mathrm{~Hz}$ and the model was big and too power demanding. Hybrid synthetic jets have also been investigated. They are similar to zero-time-mean-flow synthetic jets, but differ in the fact that a steady outflow is superposed from the nozzle, which reduces the flow-suction step. Tesar $[32,33]$ presented such an actuator for flow separation control which might have applications for turbulent flow control.

\subsection{Plasma actuators.}

In 1955, Kantrowitz presented the concept of plasma flow control. In 1983, Malik et al. controlled a boundary layer by a corona discharge. They found that when the flow velocity and the applied voltage were of $30 \mathrm{~m} / \mathrm{s}$ and $15 \mathrm{kV}$, respectively, the drag force could be reduced by $20 \%$.

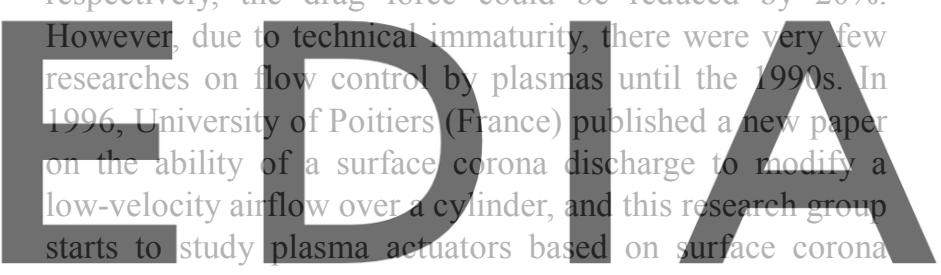
discharge as well as their effect on airflow over cylinder and

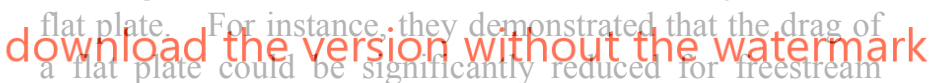
velocity up to $25 \mathrm{~m} / \mathrm{s}$ [34]. In USA, in 1998, Roth proposed an imnovative plasma actuator based on a surface dielectric barrier discharge (DBD) [35]. It is composed of two electrodes mounted on both sides of a dielectric material between which a high voltage is applied. This very simple design is still the most-used one because it enables to ignite and maintain a stable discharge whatever the environmental conditions. The control mechanism of this surface DBD is the electrohydrodynamic force that is produced inside the discharge, resulting in an electric wind based-wall jet. Typically, single DBD can produce mean force and electric wind velocity up to $1 \mathrm{mN} / \mathrm{W}$ and $7 \mathrm{~m} / \mathrm{s}$, respectively. With multi-DBD designs, velocity up to $11 \mathrm{~m} / \mathrm{s}$ has been measured and force up to $350 \mathrm{mN} / \mathrm{m}$. If the electrodes are located in the spanwise direction, then the actuation can be used to increase or to decrease the near-wall flow velocity. Moreover, with specific geometric designs, the produced flow can be strongly $3 \mathrm{D}$ inducing transverse vorticity. In fact, all the types of spatial actuation can be realized with plasma actuators simply because the electrohydrodynamic force depends only on the electrical command and the electrode 
geometry, electrodes that can be easily perfected at a laboratory scale with aluminium foils (Figure 5). For now about 15 years, several hundreds of papers have demonstrated that plasma actuators could produce a force amplitude scaling with the minimal requirements for affecting wall turbulence characteristics, controlling boundary layer and for reducing drag and noise [36].

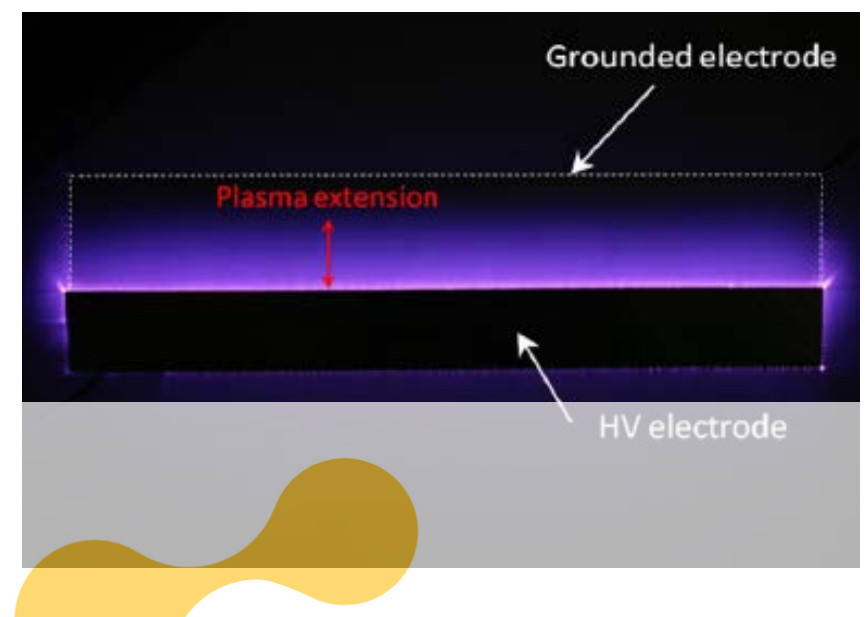

(a) Photograph of the DBD actuator
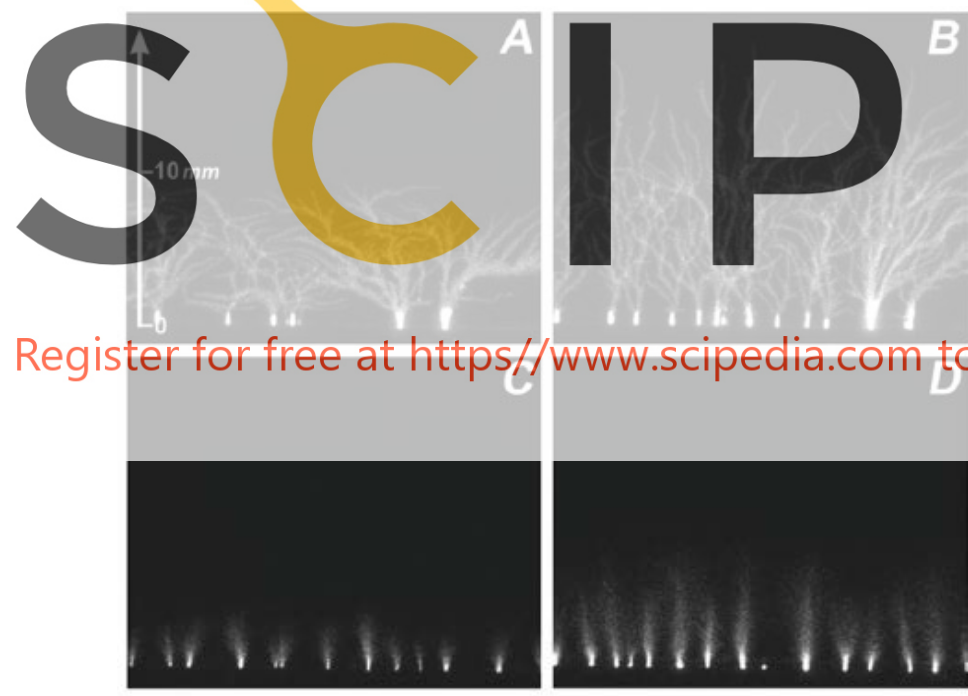

(b) Imaging of the developing plasma layer

Figure 5 Typical DBD actuator applied in turbulent boundary flow control.

However, in most cases, plasma actuators have been used to delay or cancel the flow separation, but only a few studies focused on boundary layer manipulation and skin friction drag reduction. The technical benefits of plasma actuators in terms of perturbation amplitude and frequency coupled to a quite unexplored application support any project aiming at investigating in depth the capabilities of plasma actuators to reduce skin friction drag. Indeed, among the promising applications of surface plasma actuators, the case of the manipulation of skin friction is one of the most encouraging. This type of control requires an actuator with an accurate control of the amplitude of its produced mechanical effects and a fine adjustment of the frequency content of the imposed periodic conditions. The intrinsic characteristics of an electro-mechanical converter as surface plasma actuators make them ideal candidate for open and closed-loop control of the skin friction over objects at low scale.

It is known that the turbulent boundary layer structures are quite complex with quasi-streamwise vortices caused by the elongation of the hairpin due to convection. Thus, different flow control strategies by plasma discharges have been developed in the recent years, but always at low velocity. Some of these strategies attempt at directly interact with the already formed quasi-streamwise vortices. Others approaches look at stabilization of the low-speed streaks with closed-loop strategies. As it is shown in the literature, plasma actuators can be considered as an effective way to introduce an in-plane body force compared to fluidic jet actuators. At $\operatorname{Re}_{\tau}=380$, Jukes and Choi [37] reached a 45\% skin

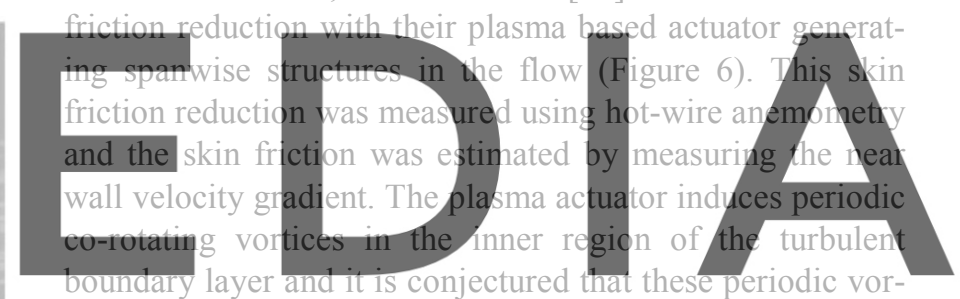

tices interact with the quasi-streamwise vortices disrupting

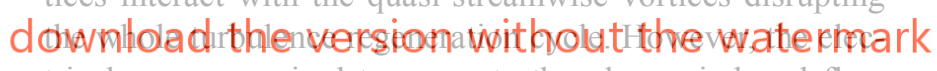

trical power required to generate the plasma induced flow oscillations was significantly greater than the net power saved by drag reduction. Another way of reducing skin friction drag is a control related to the delay of laminar to turbulent transition. Indeed, direct attenuation of existing disturbance can be achieved using plasma actuators. Such actuators can modify the velocity profile of boundary layers and thus directly alters its stability properties. A successful damping of Tollmien-Schlichting waves responsible for the early stage of transition has already been observed with surface plasma actuator [38] but the induced drag changes has not been addressed. However, because the growing rate of a laminar boundary layer is less compared to a turbulent boundary layer, the pressure drag of the body will be affected and presumably be reduced. Another method for lowering the skin friction drag of a body is the active cancellation of the 2D Tollmien-Schlichting waves before they evolve to $3 \mathrm{D}$ behaviour responsible for the formation of boundary layer vortices and turbulent spots. This concept has been investigated by producing flow perturbations at a periodic frequency similar to the frequency of the Tollmi- 
en-Schlichting waves and a phase shift of $180^{\circ}$ [39]. It was demonstrated that the natural and imposed waves interact and cancel each other leading to a laminar flow. This concept has been extended to a full-scale motorized glider [40]. By applying plasma discharges on the pressure side of a natural laminar flow wing section, microphone and hot-wire measurements have shown that a transition delay of approximately $3 \%$ chord can be achieved. A same approach was developed by Lavoie et al. [41] for in lab experiments where surface roughness are implemented on a flat plate with Blasius boundary layer to produce well-defined instability waves. A spanwise array of plasma discharges was designed to generate streaks in the boundary layer. The electro-hydrodynamic force produced by the discharge is used to inhibit the transient growth of streaks promoted by the surface roughness. It was shown that plasma is a potential technology for controlling the streaks developing in a boundary layer but nothing related to drag reduction is documented. However, as indicated previously, these experiments were conducted at low velocity.

Plasma actuators can also produce out of plane forcing conditions. For instance, Moreau et al. [42] studied plasma actuators producing wall normal jet for flow control applications. In a free-stream velocity as high as $25 \mathrm{~m} . \mathrm{s}-1$, they

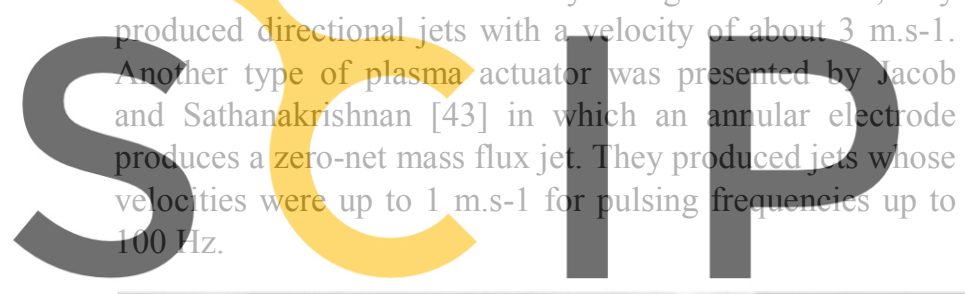

(i)

Register for free at https//www.scipedia.com to
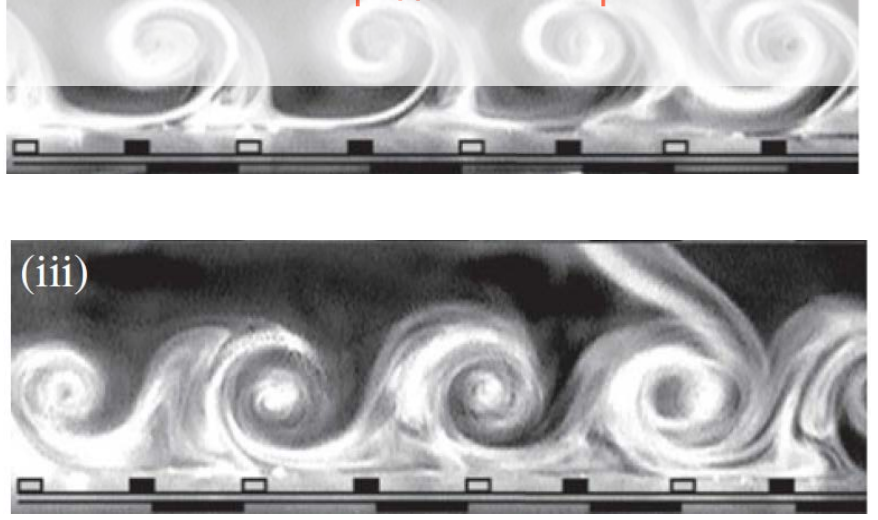

Figure 6 Typical streamwise vortical flow induced by plasma discharges in series with preferential flow direction imposed by the electrical signal.

Nowadays, although the DBD actuator structure is relatively simple, its flow control mechanism is still not fully understood. The current knowledge regarding the capability of surface plasma discharges to influence boundary layer flows in view of significant drag reduction is not yet completed. Surface plasma discharges have demonstrated their capability for stabilising the streaks in the sublayer region and they can lead to large reduction in skin friction. However, it is recognized that the electrode spacing is a critical geometrical parameters to achieve effective drag reduction. Larger improvement can be expected for well-defined geometry in particular by using panels of DBD discharges at small scale. Although many researchers in various countries have conducted a significant amount of work, to date there is no consensus on the benefits of this technology. It is certain that plasma actuators are able to address some of the deficiency of the currently available flow control technologies. Indeed, plasma actuators present some of the desired attributes including light weight, low profile, no moving parts, energy efficiency, durability, ease of use, scalability, high amplitude, wide bandwidth, and rapid response. In 2014, the AIAA Plasma dynamics and Lasers Technical Committee reports that: 'Alternating current, dielectric barrier discharge $(A C-D B D)$ plasma actuators impose a force on the flow through electro-hydrodynamic interaction. They have been used primarily to control flow separation. Their application was initially limited to low-speed flows, but has recently been extended to a higher speed regime. In recent years, these $A C-D B D$ s have been applied to

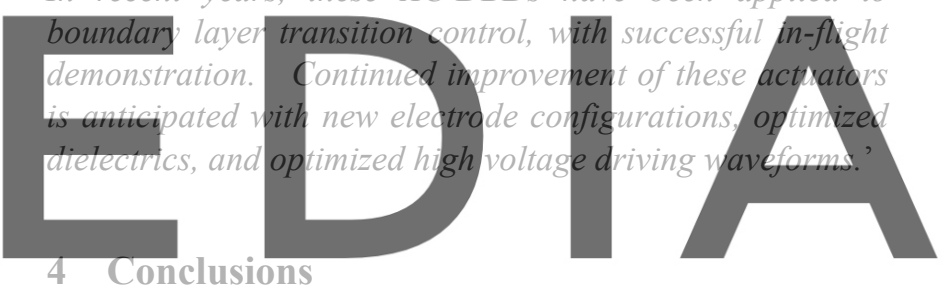

\section{download the versipn without the watermark}

understanding of the physics governing drag reduction technologies is still limited. Furthermore, the application of active control turbulent skin-friction drag reduction has seen limited success in the industrial environment.

However, given the challenging targets of the "Flightpath 2050", now is the appropriate time to investigate the potential of flow control technologies to achieve some of the ambitious ACARE environmental goals for 2020: "reducing $\mathrm{CO} 2$ emissions by $50 \%$ per passenger kilometer", "reducing NOx emissions by $80 \%$ " and "reducing perceived aircraft noise by 50\%". Reduction of $\mathrm{CO} 2$ and $\mathrm{NOx}$ emissions to the atmosphere is directly related to the aircraft fuel burn, drag and aircraft weight. Reducing drag through reduction of the aircraft weight will mitigate the environmental impacts of the emissions of the harmful gases to the atmosphere. These are the goals of the three years EU-China research project named DRAGY (Drag reduction via Turbulent Boundary Layer Flow Control) launched in April 2016. Expected outcomes of this project will result in mutual benefits for industry and scientific European as well as Chinese 
communities, in a topic of growing concern, namely drag-reduction technologies. In order to achieve these goals the following objectives are defined:

1. To identify the characteristics of flow perturbations that can be introduced into the inner and/or outer regions of the boundary layer to inhibit the drag-generating mechanisms.

2. To determine the potential drag reductions that can be achieved in representative boundary layers by targeting the turbulent structures in the inner and/or outer flow region paying particular attention to the energy cost and efficiency of the control device.

3. To develop advanced simulation methods and tools to analyse and study turbulent boundary layers at industrially relevant Reynolds numbers.

4. To demonstrate the most promising flow control concepts for turbulence manipulation and skin-friction drag reduction through experimental tests in a wind tunnel.

5. To enable the various concepts be evaluated at real aircraft level, such that an overall net energy saving

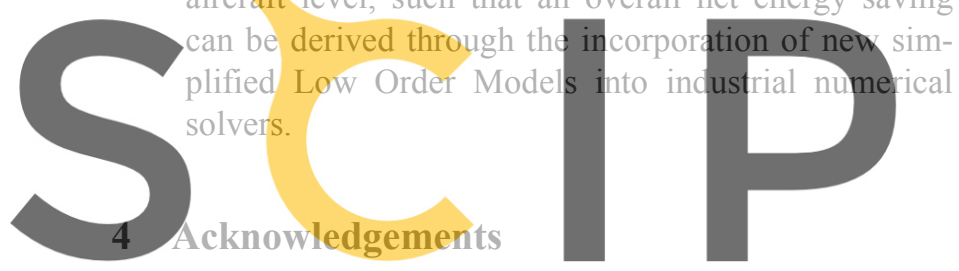

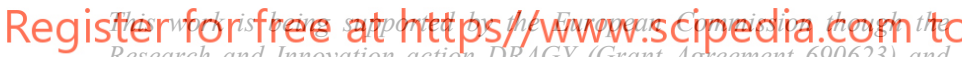
by the Ministry of Industry and Information Technology (MIIT) of the Chinese government.

The authors acknowledge all participants in DRAGY project for their contributions. In particular, special acknowledgements are dedicated to Richard Ashworth (Airbus Group Innovations), Nicolas Benard (CNRS-PPRIME), Jean-Paul Bonnet (CNRS-PPRIME), Eric Garnier (ONERA), Michael A. Leschziner (Imperial College), Shia-Hui Peng (Chalmers University), Ning Qin (University of Sheffield), Maurizio Quadrio (Politecnico di Milano), Daniel Redondo (Airbus Group Operations), Markus Ruetten (DLR) and Delille Thomas (Dassault Aviation).

We are also grateful to D. Knoerzer, EC DG Research in Research and Innovation, D. Violato, INEA, Ms. Shi Jingmin, MIIT, HUA Jun , CAE for their interest to DRAGY and their joint cooperation to set up the $D R A G Y$ project.

\section{References}

1 M. Quadrio and P. Ricco, Critical assessment of turbulent drag reduction through spanwise wall oscillations, J. Fluid Mech. 2004, 521: 251-271 2P. R. Spalart and J. Douglas McLean, Drag reduction: enticing turbu- lence, and then an industry, Phil. Trans. R. Soc. A 2011, 369: 1556-1569 3 P.S. Bernard, J.M. Thomas and R.A. Handler, Vortex dynamics and the production of Reynolds stress, J. Fluid Mech. 1993, 253: 385-419

4 A.G. Kravchenko, H. Choi, P. Moin, On the relation of near-wall streamwise vortices to wall skin friction in turbulent boundary layers, Phys. Fluids A 1993, 5: 3307-3309

5 N. Kasagi, Y. Susuki and K. Fukagata, Control of Turbulence, Parity, 2003.

6N. Kasagi, Y. Susuki and K. Fukagata, Microelectromechanical Systems-based feedback control of turbulence for skin friction reduction, Annual Reviews of Fluid Mechanics, 2009, 41: 231-251

$7 \mathrm{P}$. Viswanath, Aircraft viscous drag reduction using riblets, Progress in Aerospace Sciences, 2002, 38: 571-600

8 P. R. Spalart, J. D. McLean, Drag reduction: enticing turbulence, and then an industry, Philosophical Transactions of the Royal Society A, 2011, 369: 1556-1569

9M. J. Walsh, L. M. Weinstein, Drag and heat-transfer characteristics of small longitudinally ribbed surfaces, AIAA Journal, 1979, 17: 770-771

10 D. Bechert, M. Bruse, W. Hage, J. van derHoeven, G. Hoppe, Experiments on drag-reducing surfaces and their optimization with an adjustable geometry, J. Fluid Mech, 1997, 338: 59-87

11 P. Luchini, F. Manzo, A. Pozzi, Resistance of grooved surface to parallel flow and cross-flow, J. Fluid Mech. 1991, 228: 87-109

12 R. Garcia-Mayoral, J. Jimenez, Hydrodynamic stability and breakdown of the viscous regime over riblets, J. Fluid Mech. 2011, 678: 317-347

13 M. Sasamori, H. Mamori, K. Iwamoto, A. Muramata, Experimental study on drag-reduction effect due to sinusoidal riblets in turbulent channel flow, Experiments in Fluids, 2014, 55:1828

$14 \mathrm{M}$. Quadrio, P. Ricco and C.Viotti, Stream-wise-travelling waves of spanwise wall velocity for turbu-lent drag reduction. J. Fluid Mech. 2009, 627: 161-178
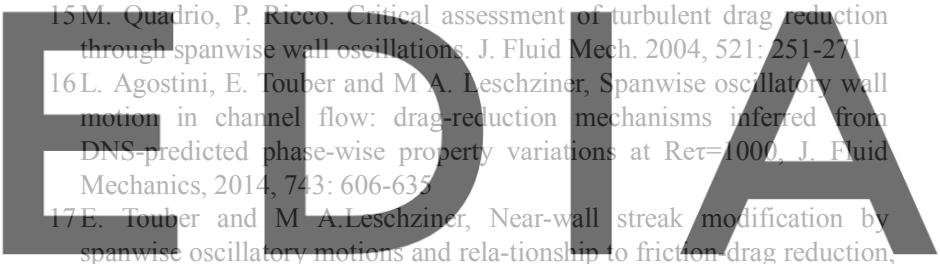

J. Fluid Mechanics, 2012, 693: 150-200

18 L. Agostini, E. Touber and M A. Leschziner. The turbulence vorticity as

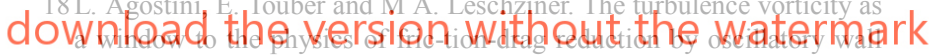

motion. Int. J. Heat and Fluid Flow, 2015, 51: 3-15

19E. Hurst, Q. Yang and Y M. Chung, The effect of Reynolds number on turbulent drag reduction by stream-wise traveliing waves, J. Fiuid Miech. 2014, 759: 28-55

20I. Marusic, R. Mathis and N. Hutchins, Predictive model for wall-bounded turbulent flow, Science, 2010, 329: 193-196

21 W. Dahm, F. Diez, Design and fabrication of unsteady electrokinetic microactuator arrays for turbulent boundary layer control. J. Micromech. Microeng. 2004, 14: 1307-1320

22 Dahm, F. Diez, Micro Electro Kinetic Actuator (MEKA) Arrays for Active sublayer control of turbulent boundary layers. AIAA, 2002, 267

23 K S. Breuer, R. Rathnasingham, Active control of turbulent boundary layers. J. Fluid Mech, 2003, 495: 209-233

24 G. Iuso, M. Cannata, Spanwise directed synthetic jets for wall turbulence control, AIAA, 2008, 4205

25 A. Pimpin, Y. Suzuki and N. Kasagi, Microelectrostrictive actuator with metal compliant electrodes for flow control applications. 17th IEEE Int. Conf. MEMS. Maastricht, 2004.

26 A. Pimpin, Y. Suzuki and N. Kasagi, Microelectrostrictive Actuator With Large Out-of-Plane deformation for flow-control application. Journal of microelectromechanical systems, 2007, 16: 753-764

27 S. Rosset, P. Niklaus, M. Dubois, M., Dadras and H. Shea, Metal Ion Implanted Compliant Electrodes in Dielectric Electroactive Polymer (EAP) Membranes, Advances in Science and Technology, 2008, 61: $18-25$

28 A. Merlen, P. Brunet, F. Zoueshtiagh, M. Pamies, E. Garnier, Microsystems for flow control and transfer: a challenge for CFD. Proceed- 
ings of 6th ICCHMT May 18-21, Guangzhou, China, 2009.

29P. Pernod and O. Ducloux, MEMS magneto-mechanical microvalves (MMMS) for aerodynamic active flow control, Journal of Magnetism and Magnetic Materials 2010, 322: 1642-1646

30 P. Mane, K. Mossi and R. Bryant, Pressure loading of Piezo Composite Unimorphs. Materials Research Society Symp. Proc. 2006.

31 Y. Liang, Y. Kuga and M. Taya, M., Design of membrane actuator based on ferromagnetic shape memory alloy composite for synthetic jet applications. Sensors and Actuators A: Physical, 2006, 125: 512-518

$32 \mathrm{~V}$. Tesar, Mechanism of pressure recovery in jet-type actuators. Sensors and Actuators A: Physical, 2009, 152: 182-191

33 V. Tesar, Z. Travnicek, J Kordik and Z. Randa, Experimental investigation of a fluidic actuator generating hybrid-synthetic jets. Sensors and Actuators A: Physical, 2007, 138: 213-220

34 L. Léger, E. Moreau, G. Touchard, Electrohydrodynamic airflow control along a flat plate by a DC surface corona discharge - Velocity profile and wall pressure measurements, AIAA, 2002, 2833.

35 J.R. Roth and D.M. Sherman, Boundary layer flow control with a One Atmosphere Uniform Glow Discharge Surface Plasma, AIAA, 1998, 328

$36 \mathrm{~N}$. Benard and E. Moreau, Electrical and mechanical characteristics of surface $\mathrm{AC}$ dielectric barrier discharge plasma actuators applied to airflow control, Experiments in Fluids, 2014, 55-1846:1-43
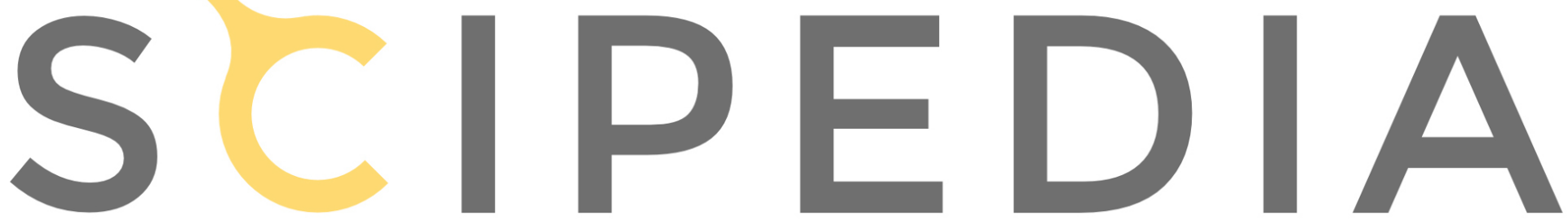

Jes and K S. Choi, Turbulent Drag Reduction by Surface Plasma through Spanwise Flow oscillation. 3rd AIAA Flow Control Conference. San Francisco, California, 2006.

38 S. Grundmann, and C. Tropea, Experimental transition delay using glow-discharge plasma actuators, Experiments in Fluids, 2007, 42: $653-657$

39S. Grundmann, and C. Tropea, Active cancellation of artificially introduced Tollmien-Schlichting waves using plasma actuators, Experiments in Fluids, 2008, 44: 795-806

40 A. Duchmann, B. Simon, C. Tropea, and S. Grundmann, Dielectric Barrier Discharge Plasma Actuators for In-Flight Transition Delay, AIAA Journal, 2014, 52: 358-367

41 R. Hanson, K. Bade, B. Belson, P. Lavoie, A. Naguib, and C. Rowley, Feedback control of slowly-varying transient growth by an array of plasma actuators, Physics of Fluids, 2014, 26

42E. Moreau et al., Aerodynamic plasma actuators: A directional micro-jet device. Thin Solid films, 2008, 516: 6660-6667

43 J D. Jacob and A. Santhanakrishnan, Flow control with plasma synthetic jet actuators. J. Phys. D: Appl. Phys., 2007, 40 